\title{
Patient Outcomes Following Interhospital Care Fragmentation: A Systematic Review
}

\author{
Katelin Snow, MD ${ }^{7}$, Karla Galaviz, MSc, $P h D^{2}$, and Sara Turbow, MD, MPH ${ }^{3}$ (iD
}

'Department of Medicine, Emory University School of Medicine, Atlanta, GA, USA; ${ }^{2}$ Hubert Department of Global Health, Emory University Rollins School of Public Health, Atlanta, GA, USA; ${ }^{3}$ Division of General Medicine and Geriatrics, Department of Medicine, Emory University School of Medicine, Atlanta, GA, USA.

\begin{abstract}
INTRODUCTION: Interhospital fragmentation of care occurs when patients are admitted to different, disconnected hospitals. It has been hypothesized that this type of care fragmentation decreases the quality of care received and increases hospital costs and healthcare utilization. This systematic review aims to synthesize the existing literature exploring the association between interhospital fragmentation of care and patient outcomes.
\end{abstract}

METHODS: MEDLINE, the Cochrane Library, EMBASE, and the Science Citation Index were systematically searched for studies published up to April 30, 2018 reporting the association between interhospital fragmentation of care and patient outcomes. We included peerreviewed observational studies conducted in adults that reported measures of association between interhospital care fragmentation and one or more of the following patient outcomes: mortality, hospital length of stay, cost, and subsequent hospital readmission.

RESULTS: Seventy-nine full texts were reviewed and 22 met inclusion criteria. Nearly all studies defined fragmentation of care as a readmission to a different hospital than the patient was previously discharged from. The strongest association reported was that between a fragmented readmission and in-hospital or short-term mortality (adjusted odds ratio range 0.95-3.62). Over half of the studies reporting length-of-stay showed longer length of stay in fragmented readmissions. All three studies that investigated healthcare utilization suggested an association between fragmented care and odds of subsequent readmission. The study populations and exposures were too heterogenous to perform a meta-analysis.

DISCUSSION: Our review suggests that fragmented hospital readmissions contribute to increased mortality, longer length-of-stay, and increased risk of readmission to the hospital.

KEY WORDS: readmission; continuity of care; fragmentation of care; patient outcomes; systematic review.

Prior Presentation: This study was presented at Emory University School of Medicine's Resident Research Day and Grady Memorial Hospital's Resident Research Day.

Electronic supplementary material The online version of this article (https://doi.org/10.1007/s11606-019-05366-z) contains supplementary material, which is available to authorized users.

Received August 6, 2019

Revised August 14, 2019

Accepted September 12, 2019

Published online October 17, 2019
$\mathrm{J}$ Gen Intern Med 35(5):1550-8

DOI: $10.1007 / \mathrm{s} 11606-019-05366-z$

๑) Society of General Internal Medicine 2019

\section{INTRODUCTION}

Increasing national emphasis has been placed on highvalue, high-quality patient care, with a focus on reducing hospital readmissions, cost, and waste. When patient care is divided among multiple organizations and providers, fragmentation of care occurs. Fragmentation of care in the outpatient setting has been associated with a range of negative patient outcomes, including increased morbidity, ${ }^{1,2}$ more duplicate medications and more drug interactions, ${ }^{3,4}$ redundant imaging tests, ${ }^{5}$ as well as more frequent admissions to the hospital. ${ }^{6-9}$ Interhospital fragmentation of care, when a patient is readmitted to a different hospital than the one they were previously discharged from, has not been widely studied, and its effects on patient outcomes are not well defined.

Among the few existing studies, interhospital care fragmentation has been reported to be associated with decreased patient satisfaction, ${ }^{10}$ longer length-of-stay, ${ }^{11-14}$ increased likelihood of discharge to a care facility, ${ }^{15}$ increased costs, ${ }^{16,17}$ and increased mortality both in the hospital and following discharge from a fragmented readmission. ${ }^{13,14,18-20}$ These studies have been conducted in diverse populations with variable definitions of care fragmentation, and no previous efforts have been made to synthesize this information.

The purpose of this review was to determine if there was an association between fragmentation of hospital care and mortality, hospital length-of-stay, cost, and readmission risk in adult patients. It is essential for health professionals, patients, and other stakeholders to understand the impact of interhospital care fragmentation on patient outcomes in order to more appropriately evaluate the value and quality of care provided, as well as the outcomes patients experience. This systematic review sheds light on this area and identifies important gaps in the evidence around the impact of care fragmentation. 


\section{METHODS}

\section{Registration, Protocol, and Disclosures}

This systematic review was registered with Prospero (CRD42018094849) and adheres to PRISMA guidelines. The authors declare that they do not have any conflicts of interest.

\section{Study Search and Selection}

We searched MEDLINE, the Cochrane Library, EMBASE, and the Science Citation Index for English articles published in peerreviewed journals through April 30, 2018. The following terms were used in our search string: "continuity of patient care," "patient readmission," "outcomes," "fragmentation of care," "fragmented care," and "discontinuity of care." A full example of our search string is included in Appendix 1. Eligible studies were cohort, case control, and cross-sectional studies, conducted in adults that reported an association between fragmentation of care and one or more of the following patient outcomes: mortality, hospital length of stay, cost, and healthcare utilization. Experimental studies, studies on pediatric patients, and qualitative studies were excluded.

One author (ST) reviewed each title and abstract for inclusion. Then two authors (ST and KS) reviewed the selected abstracts and selected studies for full text review. Then, both authors selected a set of studies to be included and disagreements were resolved by consensus. The reference list of the included studies was also hand-reviewed to identify potential additional articles. Both authors then screened these articles for inclusion.

\section{Data Extraction and Outcomes}

From the included articles, we recorded the number of patients included in each study, patient characteristics, location of the study or data source, and the definition of care fragmentation used. For each paper, we extracted the odds ratio (OR) or adjusted odds ratio (AOR) for each outcome, when reported. If OR/AOR were not available, we contacted the corresponding author of the article or calculated them from the available data. Each author was contacted up to 3 times over a 4-week period via e-mail. Given that the patient outcomes were heterogeneous, we also abstracted additional data based on the specific outcome; for instance, for studies examining mortality, the mortality follow-up period was recorded. This was not extracted for the studies examining length-of-stay or cost.

\section{Risk of Bias Assessment}

We used the Newcastle-Ottawa Scale to assess the quality and risk of bias in each study. ${ }^{21}$ The scale includes eight questions that address participant selection, the comparability of the cohorts and outcomes. For the selection domain, we assessed if the exposed cohort was representative of the study's population (1 star) or not representative (0 stars), whether the non-exposed cohort was drawn from the same community as the exposed cohort (1 star) or not ( 0 stars), how the exposure was ascertained (secure record or interview 1 star, written self-report, no description; or other 0 stars), and if it was demonstrated that the outcome of interest was not present at the start of the study (yes 1 star; no 0 stars). Next, we examined whether the study controlled for age, sex, and marital status (1 star) and/or other factors (1 star), or if the cohorts were not comparable based on the design or analysis plan of the research (0 stars). Finally, we evaluated if the outcome was assessed by independent blind assessment or record linkage (1 star), or self-report/other (0 stars), if time to follow-up was sufficient for the outcome to occur (yes 1 star; no 0 stars), and how adequate the follow-up of cohorts was - complete (1 star), less than $20 \%$ lost to follow-up/those lost to follow up were not different from those followed (1 star), or follow up rate less than $80 \%$, no description of those lost, or no statement (0 stars). The total number of stars was counted: a "good" quality study had 34 stars for selection, 1-2 stars in comparability, and 2-3 stars in outcome/exposure, a "fair" study had 2 stars for selection, 1-2 stars for comparability, and 2-3 stars in outcome/exposure, and a "poor" quality study had $0-1$ star in selection domain or 0 stars in comparability or $0-1$ stars in outcome/exposure.

Due to heterogenous study populations and a small number of articles for several outcomes, we decided to not pool the results for each outcome in a meta-analysis; additional details are provided in the "Results" section.

\section{RESULTS}

\section{Study Characteristics}

We identified 4682 unique abstracts from our literature search. Of these, 79 full texts were reviewed and 16 were included (Fig. 1). The most common reasons for exclusion were that studies analyzed outpatient continuity of care or fragmentation between physicians or emergency rooms $(n=4592)$, and that studies did not assess one of the patient outcomes of interest $(n=4056)$. The most common reason for exclusion of full-text articles reviewed was that they did not assess inpatient fragmentation $(n=30)$. Six additional articles were identified in the references of included articles and were also included in the review.

Of the 22 included studies, all but one were retrospective observational studies using secondary data. Eighteen studies reported mortality, 6 reported hospital length of stay, 4 reported hospital cost, and 3 reported hospital readmission rates. Some studies were included in multiple groups if they evaluated multiple outcomes. Duration of study follow-up ranged from 30 days to 9 years. Nineteen of the studies were in the USA, 2 in Canada, and 1 in Israel. Characteristics of each study are included in Tables 1, 2, 3, and 4. The 18 studies that evaluated patient mortality included 4,248,826 participants with a wide array of diagnoses as described in Table 1 . Hence, we determined that study populations were too heterogenous to perform a meta-analysis for this outcome. The other outcomes were limited similarly by heterogenous study populations and a small number of articles in each category. 


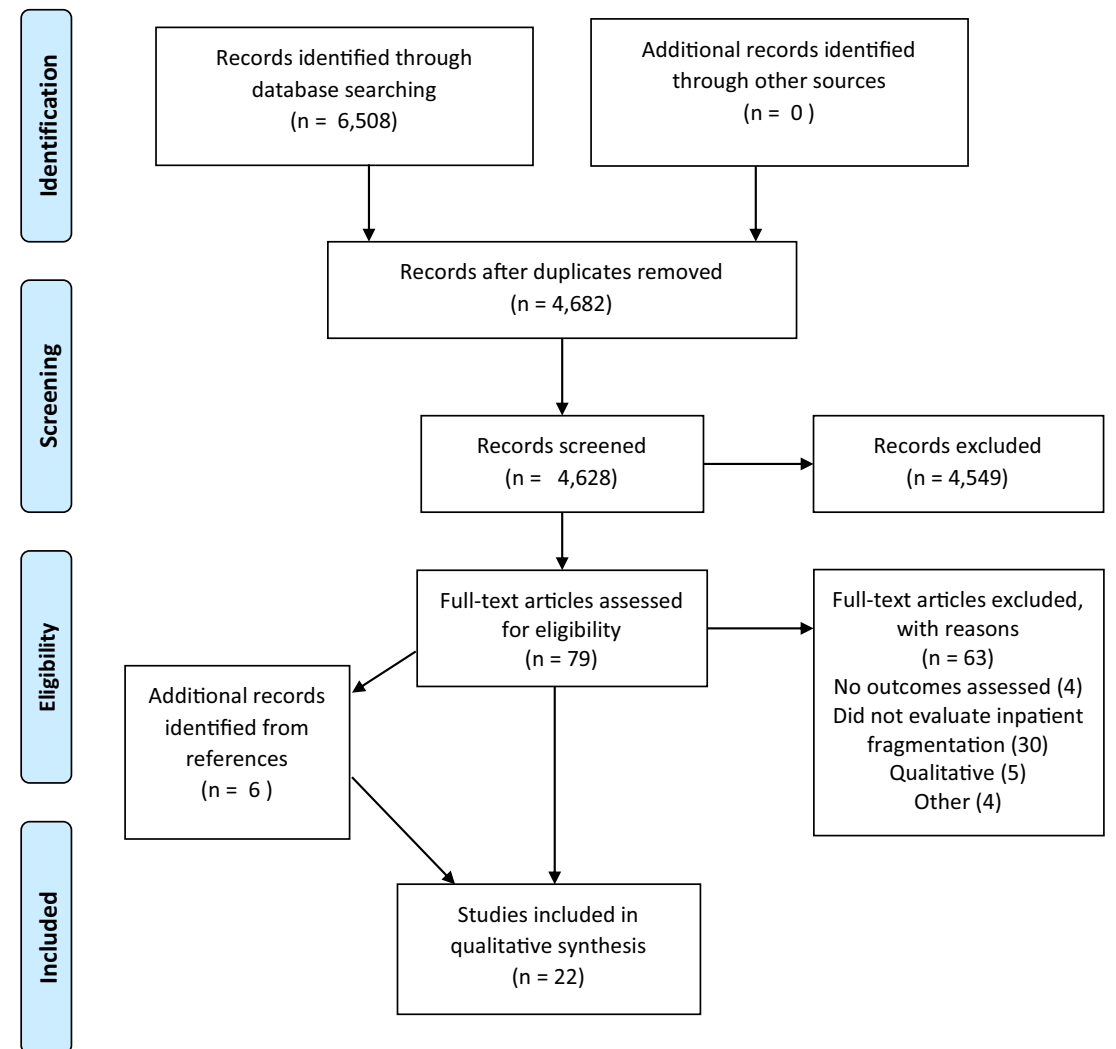

Figure 1 PRISMA flowsheet for article selection.

\section{Study Risk of Bias}

All studies generally had a clear research question, study population, and appropriate exposures and outcomes. Most of the studies $(86 \%)$ were characterized as good quality based on the Newcastle-Ottawa quality assessment tool and $14 \%$ were fair quality (Supplemental Table 1).

\section{Mortality}

Eighteen of the included articles utilized mortality as a primary endpoint (Table 1). All but two ${ }^{22,23}$ defined care fragmentation as a readmission to a different hospital than the index admission. Seven studies examined in-hospital mortality, 6 studies examined mortality 30 days post-discharge, and 9 studies examined mortality follow-up periods 90 days or longer (some studies included multiple endpoints). Of the studies examining in-hospital mortality, 4 report fragmented readmissions was associated with $10 \%$ to two times higher mortality odds (AOR 1.11-2.1). ${ }^{11}, 12,15,24$ One study had variable results depending on the factors adjusted for in analyses, ${ }^{25}$ while two did not find significant associations between fragmentation and mortality. ${ }^{26,27}$ Of the studies examining mortality post-discharge, 4 reported that fragmentation was associated with $6 \%$ to over three-fold higher odds for death within 30 days (AOR 1.06-3.62). ${ }^{20,}{ }^{28-30}$ Among 6 studies, fragmentation was associated with no increased odds to five- fold higher odds for death beyond 30 days after a fragmented readmission (AOR 1.0-5.66). ${ }^{18,19,22,23,30,31}$

Overall, 11 studies looked at mortality following a fragmented postoperative readmission, and 7 of these included cancer-related surgeries. Among the 5 studies that included only patients with cancer, ${ }^{15}, 25,30-323$ showed post-operative fragmentation associated with $18 \%$ to fivefold higher mortality odds (AOR 1.18-5.66). In 4 studies that included both cancer-related and non-cancer-related surgeries, ${ }^{19}, 20,26,27$ only 2 found post-operative care fragmentation was associated with increased odds of mortality, but the increase was notable: 50 to $75 \%$ higher mortality odds (OR 1.57-1.75).

\section{Length of Stay}

Six of the included articles used length-of-stay(LOS) as an outcome (Table 2). All defined care fragmentation as a readmission to a different hospital than the index admission. The studies varied widely in how they reported LOS, with some reporting median or mean LOS and others reporting the hazard ratio for discharge on a given day. One study reported a longer mean LOS of less than 1 day in fragmented readmissions versus non-fragmented readmissions. (11.6 days vs. 11.0 days), ${ }^{12}$ and 2 studies reported a hazard ratio of 10 $15 \%$ less for discharge on a given day in fragmented readmissions (HR 0.90-0.85). ${ }^{11,33}$ 
Table 1 Studies with Mortality as an Outcome

\begin{tabular}{|c|c|c|c|c|c|c|c|}
\hline Source & Location & $\begin{array}{l}\text { Number } \\
\text { of } \\
\text { patients }\end{array}$ & Study population & $\begin{array}{l}\text { Definition of } \\
\text { care } \\
\text { fragmentation }\end{array}$ & $\begin{array}{l}\text { Mortality } \\
\text { follow-up } \\
\text { period }\end{array}$ & $\begin{array}{l}\text { Ottawa } \\
\text { bias } \\
\text { assessment }\end{array}$ & Findings \\
\hline Jia $2007^{22}$ & Florida & 1818 & $\begin{array}{l}\text { Veterans Health } \\
\text { Administration } \\
\text { (VHA) patients } \\
\text { diagnosed with } \\
\text { acute stroke }\end{array}$ & $\begin{array}{l}\text { VHA + } \\
\text { Medicare, } \\
\text { VHA + } \\
\text { Medicaid, Triple } \\
\text { (VHA + } \\
\text { Medicare and } \\
\text { Medicaid) }\end{array}$ & $\begin{array}{l}1 \text { year after } \\
\text { discharge }\end{array}$ & Fair & $\begin{array}{l}\text { VHA-Medicare } \\
\text { AOR 1.6 (1.0-2.4) } \\
\text { VHA-Medicaid } \\
\text { AOR 2.8 }(1.0-7.4) \\
\text { Triple AOR } 1.0 \\
(0.5-2.0)\end{array}$ \\
\hline $\begin{array}{l}\text { Wolinsky } \\
2007^{23}\end{array}$ & USA & 1522 & Male veterans $>70$ & $\begin{array}{l}\text { Veterans who } \\
\text { self-reported use } \\
\text { of both VHA } \\
\text { and Medicare } \\
\text { (dual use) for in- } \\
\text { patient hospitali- } \\
\text { zations }\end{array}$ & 9-year mortality & Fair & $\begin{array}{l}\text { AHR 1.56 } \\
(1.12-2.17)\end{array}$ \\
\hline $\begin{array}{l}\text { Staples } \\
2014^{24}\end{array}$ & Toronto, Canada & 198,149 & $\begin{array}{l}\text { All adult patients } \\
\text { readmitted to one of } \\
21 \text { acute care } \\
\text { hospitals in the } \\
\text { Toronto area }\end{array}$ & $\begin{array}{l}\text { Readmission to a } \\
\text { hospital different } \\
\text { than the one they } \\
\text { were discharged } \\
\text { from }\end{array}$ & 30 days & Good & $\begin{array}{l}\text { OR } 1.26 \\
(1.23-1.30) \\
\text { AOR } 1.06 \\
(1.02-1.10)\end{array}$ \\
\hline $\begin{array}{l}\text { Saunders } \\
2014^{25}\end{array}$ & USA & 6752 & $\begin{array}{l}\text { Medicare patients } \\
\text { undergoing } \\
\text { abdominal aortic } \\
\text { aneurysm (AAA) } \\
\text { repair }\end{array}$ & $\begin{array}{l}\text { Readmission to a } \\
\text { different hospital } \\
\text { than the one } \\
\text { where the } \\
\text { surgery was } \\
\text { performed }\end{array}$ & $\begin{array}{l}\text { In-hospital and } \\
\text { 30-day mortality }\end{array}$ & Good & $\begin{array}{l}\text { In-hospital: not } \\
\text { reported (per data } \\
\text { use agreement) but } \\
\text { non-significant } \\
30 \text { days: OR } 0.95 \\
(0.56-1.62)\end{array}$ \\
\hline $\begin{array}{l}\text { Glebova } \\
2014^{26}\end{array}$ & Maryland & 115 & $\begin{array}{l}\text { Patients undergoing } \\
\text { thoracic and } \\
\text { thoracoabdominal } \\
\text { aneurysm repair, } \\
\text { 2002-2013 }\end{array}$ & $\begin{array}{l}\text { Readmission to a } \\
\text { different hospital } \\
\text { than the one } \\
\text { where the } \\
\text { surgery was } \\
\text { performed }\end{array}$ & $\begin{array}{l}\text { Mortality during } \\
\text { readmission, at } \\
30 \text { days, } 1 \text { year, } \\
5 \text { years, until } \\
\text { last follow-up }\end{array}$ & Good & $\begin{array}{l}\text { Non-fragmented vs. } \\
\text { fragmented } \\
\text { During } \\
\text { readmission: } 14 \% \\
\text { (1) vs. } 0 \\
30 \text { days: } 0 \text { vs. } 0 \\
1 \text { year: } 43 \%(3) \text { vs. } \\
8 \%(2)(p=0.05) \\
\text { At final follow-up: } \\
43 \%(3) \text { vs. } 15 \% \\
\text { (4) }(p=0.11)\end{array}$ \\
\hline Tsai $2015^{27}$ & USA (Medicare) & 93,062 & $\begin{array}{l}\text { Patients with } \\
\text { coronary artery } \\
\text { bypass graft, } \\
\text { pulmonary } \\
\text { lobectomy, } \\
\text { endovascular } \\
\text { aneurysm repair, } \\
\text { open AAA repair, } \\
\text { colectomy, total hip } \\
\text { replacement with a } \\
\text { 30-day readmission }\end{array}$ & $\begin{array}{l}\text { Readmission to a } \\
\text { different hospital } \\
\text { than the one } \\
\text { where the } \\
\text { surgery was } \\
\text { performed }\end{array}$ & 30-day mortality & Good & $\begin{array}{l}\text { Risk and hospital } \\
\text { adjusted: OR } 1.41 \\
\text { (1.31-1.52) } \\
\text { Distance-adjusted: } \\
\text { OR 1.48 } \\
(1.37-1.59)\end{array}$ \\
\hline $\begin{array}{l}\text { Brooke } \\
2015^{18}\end{array}$ & USA (Medicare) & $1,111,046$ & $\begin{array}{l}\text { Patients undergoing } \\
\text { one of } 12 \text { major } \\
\text { operations* } \\
\text { readmitted within } \\
30 \text { days, 2001-2011 }\end{array}$ & $\begin{array}{l}\text { Readmission to a } \\
\text { different hospital } \\
\text { than the one } \\
\text { where the } \\
\text { surgery was } \\
\text { performed }\end{array}$ & 90-day mortality & Good & $\begin{array}{l}\text { Pooled AOR (Ref: } \\
\text { fragmented } \\
\text { readmission) } 0.74 \\
(0.66-0.83)\end{array}$ \\
\hline Pak $2015^{28}$ & New York State & 2338 & $\begin{array}{l}\text { Patients discharged } \\
\text { from one of } 100 \\
\text { New York State } \\
\text { Hospitals following } \\
\text { radical cystectomy } \\
\text { between } 2009 \text { and } \\
2012 \text { with } 30 \text { or } 90 \text { - } \\
\text { day readmissions }\end{array}$ & $\begin{array}{l}\text { Readmission to a } \\
\text { different hospital } \\
\text { than the one } \\
\text { where the } \\
\text { surgery was } \\
\text { performed }\end{array}$ & $\begin{array}{l}30 \text { - and } 90 \text {-day } \\
\text { mortality }\end{array}$ & Good & $\begin{array}{l}30 \text { days OR } 3.62 \\
(1.52-8.57) \\
90 \text { days OR } 5.66 \\
(2.63-12.2)\end{array}$ \\
\hline Luu $2016^{29}$ & $\begin{array}{l}\text { USA } \\
\text { (Surveillance } \\
\text { Epidemiology, } \\
\text { and End Results } \\
\text { Program } \\
\text { [SEER]- } \\
\text { Medicare) }\end{array}$ & 3399 & $\begin{array}{l}\text { Patients who had } \\
\text { colon cancer surgery } \\
\text { who were readmitted } \\
\text { within } 30 \text { days of } \\
\text { discharge, } \\
2000-2009\end{array}$ & $\begin{array}{l}\text { Readmission to a } \\
\text { different hospital } \\
\text { than the one } \\
\text { where the } \\
\text { surgery was } \\
\text { performed }\end{array}$ & $\begin{array}{l}\text { Long-term } \\
\text { mortality } \\
\text { colon-cancer } \\
\text { specific } \\
\text { mortality } \\
90 \text {-day mortality } \\
\text { (all-cause and } \\
\text { cancer-specific) }\end{array}$ & Good & $\begin{array}{l}\text { Long-term } \\
\text { mortality: HR } 1.04 \\
\text { ( } 95 \% \text { CI } 0.87-1.12 \text { ) } \\
\text { Colon } \\
\text { cancer-specific } \\
\text { mortality: } 1.09 \\
\text { ( } 95 \% \text { CI } 0.77-1.51 \text { ) }\end{array}$ \\
\hline
\end{tabular}


Table 1. (continued)

\begin{tabular}{|c|c|c|c|c|c|c|c|}
\hline Source & Location & $\begin{array}{l}\text { Number } \\
\text { of } \\
\text { patients }\end{array}$ & Study population & $\begin{array}{l}\text { Definition of } \\
\text { care } \\
\text { fragmentation }\end{array}$ & $\begin{array}{l}\text { Mortality } \\
\text { follow-up } \\
\text { period }\end{array}$ & $\begin{array}{l}\text { Ottawa } \\
\text { bias } \\
\text { assessment }\end{array}$ & Findings \\
\hline & & & & & & & $\begin{array}{l}\text { 90-day mortality: } \\
1.18(95 \% \text { CI } \\
1.02-1.38)\end{array}$ \\
\hline $\begin{array}{l}\text { Mays } \\
2016^{30}\end{array}$ & Chicago, IL & 780 & $\begin{array}{l}\text { Patients with } \\
\text { recurrent diabetic } \\
\text { ketoacidosis, } \\
2006-2012\end{array}$ & $\begin{array}{l}\text { Readmission to a } \\
\text { different hospital } \\
\text { than the patient } \\
\text { was discharged } \\
\text { from }\end{array}$ & $\begin{array}{l}\text { Mortality during } \\
\text { study period }\end{array}$ & Good & $\begin{array}{l}\text { NR but non- } \\
\text { significant in } \\
\text { adjusted models }\end{array}$ \\
\hline $\begin{array}{l}\text { Zheng } \\
2016^{31}\end{array}$ & $\begin{array}{l}\text { California (State } \\
\text { Inpatient } \\
\text { Database [SID]) }\end{array}$ & 9233 & $\begin{array}{l}\text { Patients with major } \\
\text { cancer surgery† } \\
\text { readmitted within } \\
30 \text { days, 2004-2011 }\end{array}$ & $\begin{array}{l}\text { Readmission to a } \\
\text { different hospital } \\
\text { than the one } \\
\text { where the } \\
\text { surgery was } \\
\text { performed }\end{array}$ & $\begin{array}{l}\text { In-hospital } \\
\text { mortality }\end{array}$ & Good & $\begin{array}{l}\text { Adjusted for patient } \\
\text { and hospital } \\
\text { characteristics: } \\
1.31(1.03-1.66) \\
\text { Adjusted for } \\
\text { diagnoses: } 1.24 \\
(0.98-1.58)\end{array}$ \\
\hline $\begin{array}{l}\text { Kothari } \\
2016^{20}\end{array}$ & $\begin{array}{l}\text { California and } \\
\text { Florida }\end{array}$ & 2996 & $\begin{array}{l}\text { Patients who } \\
\text { underwent orthotopic } \\
\text { liver transplant } \\
\text { readmitted within the } \\
\text { first year, 2006-2011 }\end{array}$ & $\begin{array}{l}\text { Readmission to a } \\
\text { hospital other } \\
\text { than where } \\
\text { surgery was } \\
\text { performed }\end{array}$ & $\begin{array}{l}\text { 30-day } \\
\text { mortality after } \\
\text { readmission }\end{array}$ & Good & $\begin{array}{l}\text { OR } 1.75 \text { (1.16 to } \\
2.65)\end{array}$ \\
\hline $\begin{array}{l}\text { Justiniano } \\
2017^{19}\end{array}$ & New York State & 20,016 & $\begin{array}{l}\text { Patients readmitted } \\
\text { within } 30 \text { days, } \\
\text { 2004-2014 }\end{array}$ & $\begin{array}{l}\text { Readmission to a } \\
\text { different hospital } \\
\text { than where } \\
\text { surgery was } \\
\text { performed } \pm \\
\text { under the care of } \\
\text { a different } \\
\text { physician }\end{array}$ & $\begin{array}{l}\text { 1-year survival } \\
\text { (excluding } \\
\text { initial 30-day } \\
\text { mortality) }\end{array}$ & Good & $\begin{array}{l}\text { HR 1.57 } \\
(1.17-2.11)\end{array}$ \\
\hline $\begin{array}{l}\text { Stitzenberg } \\
2017^{32}\end{array}$ & $\begin{array}{l}\text { USA (SEER- } \\
\text { Medicare) }\end{array}$ & 7903 & $\begin{array}{l}\text { Patients with cancer: } \\
\text { who underwent } \\
\text { extirpative surgery, } \\
\text { 2001-2007 }\end{array}$ & $\begin{array}{l}\text { Readmission to a } \\
\text { different hospital } \\
\text { than the one } \\
\text { where the } \\
\text { surgery was } \\
\text { performed }\end{array}$ & 90-day mortality & Good & $\begin{array}{l}\text { HR 1.15 } \\
(1.10-1.19)\end{array}$ \\
\hline Hua $2017^{33}$ & New York State & 26,947 & $\begin{array}{l}\text { Mechanically } \\
\text { ventilated intensive } \\
\text { care unit patients } \\
\text { re-hospitalized } \\
\text { within } 30 \text { days at NY } \\
\text { state hospitals } \\
\text { between } 2008 \\
\text { and } 2013\end{array}$ & $\begin{array}{l}\text { Readmission to a } \\
\text { different hospital } \\
\text { than the patient } \\
\text { was discharged } \\
\text { from }\end{array}$ & $\begin{array}{l}\text { In-hospital } \\
\text { mortality }\end{array}$ & Good & $\begin{array}{l}\text { ARR 1.11 } \\
(1.03-1.20)\end{array}$ \\
\hline $\begin{array}{l}\text { McAlister } \\
2017^{12}\end{array}$ & Canada & 39,368 & $\begin{array}{l}\text { Patients with heart } \\
\text { failure readmitted } \\
\text { within } 30 \text { days, } \\
\text { 2004-2013 }\end{array}$ & $\begin{array}{l}\text { Readmission to a } \\
\text { different hospital } \\
\text { than the patient } \\
\text { was discharged } \\
\text { from }\end{array}$ & $\begin{array}{l}\text { In-hospital } \\
\text { mortality }\end{array}$ & Good & $\begin{array}{l}\text { OR } 0.89 \\
(0.82-0.96) \\
* \text { Reference was } \\
\text { fragmented group }\end{array}$ \\
\hline $\begin{array}{l}\text { Graboyes } \\
2017^{15}\end{array}$ & California & 561 & $\begin{array}{l}\text { Patients readmitted } \\
\text { within } 30 \text { days of a } \\
\text { head and neck cancer } \\
\text { surgery, 2008-2010 }\end{array}$ & $\begin{array}{l}\text { Readmission to a } \\
\text { hospital other } \\
\text { than where } \\
\text { surgery was } \\
\text { performed }\end{array}$ & $\begin{array}{l}\text { In-hospital } \\
\text { mortality }\end{array}$ & Good & OR $2.1(1.04-4.26)$ \\
\hline $\begin{array}{l}\text { Burke } \\
2018^{11}\end{array}$ & $\begin{array}{l}\text { USA (National } \\
\text { Readmissions } \\
\text { Database } \\
{[\text { NRD]) }}\end{array}$ & $2,722,821$ & $\begin{array}{l}\text { Patients readmitted } \\
\text { within } 30 \text { days in } \\
2013\end{array}$ & $\begin{array}{l}\text { Readmission to a } \\
\text { different hospital } \\
\text { than the patient } \\
\text { was discharged } \\
\text { from }\end{array}$ & $\begin{array}{l}\text { In-hospital } \\
\text { mortality }\end{array}$ & Good & $\begin{array}{l}\text { OR } 1.14 \\
(1.10-1.18) \\
\text { AOR } 1.21 \\
(1.17-1.25)\end{array}$ \\
\hline
\end{tabular}

*Open AAA repair, infra-inguinal arterial bypass, aorto-bifemoral bypass, coronary artery bypass surgery, esophagectomy, colectomy, pancreatectomy, cholecystectomy, ventral hernia repair, craniotomy, hip or knee replacement

tEsophagectomy, gastrectomy, pancreatectomy, hepatectomy, proctectomy, lung resection

$\ddagger$ Bladder, esophagus, lung, pancreas cancer

\section{Costs and Charges}

Four of the included studies examined cost or charges as a primary or secondary outcome (Table 3 ). These studies varied in their timeframe of measurement: 2 examined in-hospital costs or charges, 1 examined costs for a year following cancer diagnosis, and 1 examined costs for 90 days following surgery.
The differences in costs/charges between fragmented and nonfragmented readmissions ranged from $\$ 270$ to $\$ 22,000$. Only 1 found statistically significantly higher costs in patients with fragmented readmissions in adjusted models, with a median cost of a fragmented readmission of $\$ 8568$ and the median cost of a non-fragmented readmission of $\$ 8298 .{ }^{17}$ 
Table 2 Studies with LOS as an Outcome

\begin{tabular}{|c|c|c|c|c|c|c|}
\hline Reference & $\begin{array}{l}\text { Data source } \\
\text { and location }\end{array}$ & $\begin{array}{l}\text { Number of } \\
\text { patients }\end{array}$ & Study population & $\begin{array}{l}\text { Definition of care } \\
\text { fragmentation }\end{array}$ & $\begin{array}{l}\text { Ottawa bias } \\
\text { assessment }\end{array}$ & Findings \\
\hline $\begin{array}{l}\text { Galanter } \\
2013^{14}\end{array}$ & Chicago & 228,151 & $\begin{array}{l}\text { Patients admitted to } 5 \\
\text { teaching hospitals } \\
\text { 2007-2008 }\end{array}$ & $\begin{array}{l}\text { Admitted to more } \\
\text { than one hospital }\end{array}$ & Fair & $\begin{array}{l}\text { Patients admitted to more } \\
\text { than one hospital mean } \\
\text { LOS } 5.58 \pm 8.42 \\
\text { Patients with multiple } \\
\text { admissions to one } \\
\text { hospital mean LOS } \\
5.77 \pm 8.42\end{array}$ \\
\hline $\begin{array}{l}\text { Brooke } \\
2015^{18}\end{array}$ & $\begin{array}{l}\text { USA } \\
\text { (Medicare) }\end{array}$ & $1,111,046$ & $\begin{array}{l}\text { Patients undergoing one } \\
\text { of } 12 \text { major operations* } \\
\text { readmitted within } \\
30 \text { days, } 2001-2011\end{array}$ & $\begin{array}{l}\text { Readmission to a different } \\
\text { hospital than the one where } \\
\text { the surgery was performed }\end{array}$ & Good & $\begin{array}{l}\text { Fragmented vs. not } \\
\text { (mean LOS) } \\
\text { Vascular: } 6.7 \text { vs. } 8.2 \\
\text { Cardiothoracic: } 5.6 \text { vs. } \\
7.1 \\
\text { General: } 6.5 \text { vs. } 7.1 \\
\text { Neurosurgery: } 6.4 \text { v. } 7.4 \\
\text { Orthopedic: NR }\end{array}$ \\
\hline $\begin{array}{l}\text { Flaks- } \\
\text { Manov } \\
2016^{34}\end{array}$ & Israel & 27,057 & $\begin{array}{l}\text { Adult patients with state } \\
\text { insurance with at least } 1 \\
\text { readmission in } 2010\end{array}$ & $\begin{array}{l}\text { Readmission to a different } \\
\text { hospital (DHR) with or } \\
\text { without a health informa- } \\
\text { tion exchange (HIE) }\end{array}$ & Good & $\begin{array}{l}\text { DHR without HIE: HR } \\
0.90 \text { (CI } 0.86-0.94) \\
\text { DHR with HIE: } 0.85 \\
(0.79-0.91)\end{array}$ \\
\hline $\begin{array}{l}\text { McAlister } \\
2017^{12}\end{array}$ & Canada & 39,368 & $\begin{array}{l}\text { Patients with heart } \\
\text { failure, } 2004-2013 \\
\text { readmitted within } \\
30 \text { days }\end{array}$ & $\begin{array}{l}\text { Readmission to a different } \\
\text { hospital than the patient } \\
\text { was discharged from }\end{array}$ & Good & $\begin{array}{l}\text { Fragmented mean LOS } \\
11.6(11.3-12.0) n=6597 \\
\text { Non-fragmented mean } \\
\text { LOS } 11.0(10.1-12.0) \\
n=32771\end{array}$ \\
\hline $\begin{array}{l}\text { Graboyes } \\
2017^{15}\end{array}$ & California & 561 & $\begin{array}{l}\text { Patients readmitted } \\
\text { within } 30 \text { days of a head } \\
\text { and neck cancer surgery, } \\
2008-2010\end{array}$ & $\begin{array}{l}\text { Readmission to a hospital } \\
\text { other than where surgery } \\
\text { was performed }\end{array}$ & Good & $\begin{array}{l}\text { Median LOS fragmented } \\
\text { v. not } 4 \text { vs. } 5\end{array}$ \\
\hline $\begin{array}{l}\text { Burke } \\
2018^{11}\end{array}$ & USA (NRD) & $2,722,821$ & $\begin{array}{l}\text { Patients readmitted } \\
\text { within } 30 \text { days in } 2013\end{array}$ & $\begin{array}{l}\text { Readmission to a different } \\
\text { hospital than the patient } \\
\text { was discharged from }\end{array}$ & Good & $\begin{array}{l}\text { Readmission to non- } \\
\text { index adjusted HR } \\
0.87(0.86-0.88)\end{array}$ \\
\hline
\end{tabular}

*Open AAA repair, infra-inguinal arterial bypass, aorto-bifemoral bypass, coronary artery bypass surgery, esophagectomy, colectomy, pancreatectomy, cholecystectomy, ventral hernia repair, craniotomy, hip or knee replacement

Table 3 Studies with Cost/Charges as an Outcome

\begin{tabular}{|c|c|c|c|c|c|c|c|}
\hline Reference & $\begin{array}{l}\text { Data } \\
\text { source } \\
\text { and } \\
\text { location }\end{array}$ & $\begin{array}{l}\text { Number } \\
\text { of } \\
\text { patients }\end{array}$ & Study population & $\begin{array}{l}\text { Definition of care } \\
\text { fragmentation }\end{array}$ & $\begin{array}{l}\text { Ottawa } \\
\text { bias } \\
\text { assessment }\end{array}$ & $\begin{array}{l}\text { Outcome } \\
\text { assessed }\end{array}$ & Findings \\
\hline $\begin{array}{l}\text { Glebova } \\
2014^{26}\end{array}$ & Maryland & 115 & $\begin{array}{l}\text { Patients undergoing } \\
\text { thoracic and } \\
\text { thoracoabdominal } \\
\text { aneurysm repair, 2002- } \\
2013\end{array}$ & $\begin{array}{l}\text { Readmission to a } \\
\text { different hospital } \\
\text { than the one where } \\
\text { the surgery was } \\
\text { performed }\end{array}$ & Good & $\begin{array}{l}\text { Total } \\
\text { charges for } \\
\text { readmission }\end{array}$ & $\begin{array}{l}\text { Fragmented } \\
\text { total: } 20,000 \pm 4400 \\
\text { Non-fragmented } \\
\text { total: } 42,000 \pm 8800\end{array}$ \\
\hline Luu $2016^{29}$ & $\begin{array}{l}\text { USA } \\
\text { (SEER- } \\
\text { Medicare) }\end{array}$ & 3,399 & $\begin{array}{l}\text { Patients who had colon } \\
\text { cancer surgery who } \\
\text { were readmitted within } \\
30 \text { days of discharge, } \\
2000-2009\end{array}$ & $\begin{array}{l}\text { Readmission to a } \\
\text { different hospital } \\
\text { than the one where } \\
\text { the surgery was } \\
\text { performed }\end{array}$ & Good & $\begin{array}{l}\text { Cost of care } \\
\text { for up to } \\
1 \text { year after } \\
\text { index } \\
\text { admission }\end{array}$ & $\begin{array}{l}\text { Difference } \\
\$ 8405(95 \% \mathrm{CI} \\
-\$ 4202 \text { to } \$ 23,114)\end{array}$ \\
\hline $\begin{array}{l}\text { Stitzenberg } \\
2017^{32}\end{array}$ & $\begin{array}{l}\text { USA } \\
\text { (SEER- } \\
\text { Medicare) }\end{array}$ & 7903 & $\begin{array}{l}\text { Patients with cancer* } \\
\text { who underwent } \\
\text { extirpative surgery, } \\
\text { 2001-2007 }\end{array}$ & $\begin{array}{l}\text { Readmission to a } \\
\text { different hospital } \\
\text { than the one where } \\
\text { the surgery was } \\
\text { performed }\end{array}$ & Good & $\begin{array}{l}\text { Total costs } \\
\text { incurred in } \\
\text { the } 90 \text { days } \\
\text { post-index } \\
\text { discharge }\end{array}$ & $\begin{array}{l}\text { Fragmented v. not } \\
\text { Medical diagnoses: } \\
\$ 37,806 \pm \$ 55,730 \text { vs. } \\
\$ 36,052 \pm \$ 37,541 \\
\text { Surgical diagnoses: } \\
\$ 50,465 \pm \$ 75,710 \text { vs. } \\
\$ 56,559 \pm \$ 62,438\end{array}$ \\
\hline $\begin{array}{l}\text { Parreco } \\
2017^{17}\end{array}$ & $\begin{array}{l}\text { USA } \\
\text { (NRD) }\end{array}$ & 110,854 & $\begin{array}{l}\text { Patients admitted with a } \\
\text { principal diagnosis of } \\
\text { trauma in 2013-2014 }\end{array}$ & $\begin{array}{l}\text { Readmission to a } \\
\text { different hospital } \\
\text { than the one they } \\
\text { were previously } \\
\text { discharged from }\end{array}$ & Good & $\begin{array}{l}\text { Total cost of } \\
\text { readmission }\end{array}$ & $\begin{array}{l}\text { Fragmented (median) } \\
\$ 8568 \text { (IQR } \\
\$ 4935-\$ 16,078) \\
\text { Non-fragmented } \\
\text { (median) } \\
\$ 8298 \text { (IQR } \\
\$ 4899-\$ 14,911)\end{array}$ \\
\hline
\end{tabular}


Table 4 Studies with Readmissions as an Outcome

\begin{tabular}{|c|c|c|c|c|c|c|}
\hline Reference & $\begin{array}{l}\text { Data source } \\
\text { and location }\end{array}$ & $\begin{array}{l}\text { Number of } \\
\text { patients }\end{array}$ & Study population & $\begin{array}{l}\text { Definition of care } \\
\text { fragmentation }\end{array}$ & $\begin{array}{l}\text { Ottawa bias } \\
\text { assessment }\end{array}$ & $\begin{array}{l}\text { AOR/OR for } \\
\text { rehospitalization }\end{array}$ \\
\hline Jia $2007^{22}$ & Florida & 1818 & $\begin{array}{l}\text { VA patients diagnosed } \\
\text { with acute stroke }\end{array}$ & $\begin{array}{l}\text { VHA + Medicare, VHA + } \\
\text { Medicaid, Triple (VHA + } \\
\text { Medicare and Medicaid) }\end{array}$ & Good & $\begin{array}{l}\text { VHA + Medicare: AOR } \\
1.5(1.1-1.9) \\
\text { VHA + Medicaid: } \\
\text { AOR 2.3 (1.2-4.5) } \\
\text { Triple: AOR } 13.6 \\
(6.1-30.1)\end{array}$ \\
\hline $\begin{array}{l}\text { Axon } \\
2016^{35}\end{array}$ & USA & 13,977 & $\begin{array}{l}\text { Veterans with heart } \\
\text { failure hospitalized at the } \\
\text { VA or non-VA facilities, } \\
2007-2011\end{array}$ & Dual use & Good & AOR $1.93(1.85-2.01)$ \\
\hline $\begin{array}{l}\text { Zheng } \\
2016^{31}\end{array}$ & $\begin{array}{l}\text { California } \\
\text { (SID) }\end{array}$ & 9233 & $\begin{array}{l}\text { Patients with major } \\
\text { cancer surgery* } \\
\text { readmitted within } \\
30 \text { days }\end{array}$ & $\begin{array}{l}\text { Readmission to a different } \\
\text { hospital than the one where } \\
\text { the surgery was performed }\end{array}$ & Good & $\begin{array}{l}\text { Adjusted for patient and } \\
\text { hospital characteristics: } \\
1.17(1.03-1.34) \\
\text { Adjusted for patient } \\
\text { characteristics: } 1.16 \\
(1.02-1 / 32)\end{array}$ \\
\hline
\end{tabular}

*Esophagectomy, gastrectomy, pancreatectomy, hepatectomy, proctectomy, lung resection

\section{Hospital Readmission}

Three papers examined the association of care fragmentation and hospital readmission (Table 4). They found that patients with fragmented readmissions were between $16 \%$ and twice as likely to experience a third admission than patients who did not experience interhospital care fragmentation (AOR 1.16-2.3). ${ }^{23,} 25,34$ Notably, one subgroup who accessed care at the VA and also held Medicare and Medicaid insurance was reported to have an AOR of 13.6 (95\% CI 6.1-30.1) for a third admission following a fragmented readmission. ${ }^{23}$

\section{DISCUSSION}

In this systematic review, we analyzed 22 studies examining patient outcomes during and following a fragmented hospital readmission. Overall, 11 of the 18 studies that examined patient mortality found increased odds of mortality during or following a fragmented readmission, 1 had mixed results, and 6 did not show a difference in the odds of mortality. Three of the 6 studies reporting length-of-stay found a longer length-ofstay or decreased hazard ratio for discharge in fragmented readmissions. Among studies examining cost/charges, 1 study reported higher costs during a fragmented readmission, 1 had mixed results, and 2 reported higher costs/charges during or after non-fragmented readmissions. Finally, all three papers that investigated the odds of a third hospital readmission following a fragmented readmission found higher odds of a third admission in patients who experienced fragmentation, compared to patients who had a non-fragmented readmission. Overall, this systematic review suggests that fragmented hospital readmissions contribute to increased mortality, longer length-of-stay, and increased risk of future readmission to the hospital.
Our systematic review adds to the previous reviews of the association between continuity of care and patient outcomes. ${ }^{2}$, ${ }^{35}$ Previous reviews have focused on outpatients only or on continuity during transitions of care (i.e., inpatient to outpatient). Van Walraven et al. ${ }^{2}$ performed a systematic review of studies examining continuity of care, healthcare use, and patient satisfaction, primarily focused on continuity following hospital discharge and in the outpatient setting. They found that there were significant associations between improved provider continuity, decreased healthcare use, and increased patient satisfaction. Our review is the first to synthesize the literature on fragmented hospital readmissions and adds to the existing literature by including papers that address different types of continuity. ${ }^{23,33}$

Our study is subject to limitations. Our definition of interhospital care fragmentation was narrow in an effort to make studies as comparable as possible, which may have excluded some studies looking at other types of hospital-based fragmentation (i.e., fragmentation across providers in a hospital or interhospital transfers). Notably, many of the included studies examined very specific populations (i.e., postoperative from cancer surgery), making the results less generalizable. Finally, because of the retrospective nature of most of the included studies, we are unable to know whether fragmentation was the cause or an effect of a patient's poor outcomes. ${ }^{2}$ This is especially true regarding hospital readmissions - if the patient had a poor experience during their index admission, they may actively seek care elsewhere, leading to a fragmented readmission. If they remain dissatisfied following the readmission or their health has not improved, they may seek care at yet another hospital.

In conclusion, this systematic review suggests that interhospital care fragmentation may be a contributor to increased mortality, longer lengths-of-stay, and readmissions. These results support the need for improved care coordination and should increase provider awareness of the role of interhospital 
care fragmentation on our patients and hospitals. Interhospital care fragmentation should be considered when designing interventions to reduce duplicative care and waste, and when evaluating provider and hospital outcomes.

Corresponding Author: Sara Turbow, MD, MPH; Division of General Medicine and Geriatrics, Department of MedicineEmory University School of Medicine, Atlanta, GA, USA (e-mail: sara.turbow@emory. edu).

Author's Contribution No one contributed to the article who is not listed as an author.

\section{Compliance with Ethical Standards:}

Conflict of Interest: The authors declare that they do not have a conflict of interest.

\section{REFERENCES}

1. Weir DL, McAlister FA, Majumdar SR, Eurich DT. The interplay between continuity of care, multimorbidity, and adverse events in patients with diabetes. Med Care 2016;54(4):386-393. https://doi.org/ 10.1097/MLR.0000000000000493.

2. Van Walraven C, Oake N, Jennings A, Forster AJ. The association between continuity of care and outcomes: a systematic and critical review. J Eval Clin Pract. 2010;16(5):947-956. https://doi.org/10.1111/ j.1365-2753.2009.01235.x.

3. Guo J-Y, Chou Y-J, Pu C, Pu C. Effect of continuity of care on drug-drug interactions. Med Care. 2017;55(8):744-751

4. Lai Y-R, Yang Y-S, Tsai M-L, et al. Impact of potentially inappropriate medication and continuity of care in a sample of Taiwan elderly patients with diabetes mellitus who have also experienced heart failure. Geriatr Gerontol Int. 2015;16(10):1117-1126. https://doi.org/10.1111/ggi. 12606.

5. Bekelis K, Roberts DW, Zhou W, Skinner J. Fragmentation of care and the use of head computed tomography in patients with ischemic stroke. Circ Cardiovasc Qual Outcomes. 2014;7(3):430-436. https://doi.org/10. 1161/CIRCOUTCOMES.

6. Nyweide DJ, Anthony DL, Bynum JP, et al. Continuity of care and the risk of preventable hospitalization in older adults. JAMA Intern Med. 2013;173(20):1879-1885. https://doi.org/10.1001/jamainternmed. 2013.10059.

7. Kao Y-H, Wu S-C.STROBE-compliant article: is continuity of care associated with avoidable hospitalization in older asthmatic patients? Medicine. 2016;95(38):e4948-7. https://doi.org/10.1097/MD. 0000000000004948.

8. Lin I-P, Wu S-C. Effects of long-term high continuity of care on avoidable hospitalizations of chronic obstructive pulmonary disease patients. Health Policy. 2017;121(9):1001-1007. https://doi.org/10.1016/j. healthpol.2017.06.010.

9. Missios S, Bekelis K. Outpatient continuity of care and 30-day readmission after spine surgery. Spine J. 2016;16(11):1309-1314. https://doi.org/10.1016/j.spinee.2016.06.012.

10. Fan VS, Burman M, McDonell MB, Fihn SD. Continuity of care and other determinants of patient satisfaction with primary care. JGIM. 2005;20(3):226-233. https://doi.org/10.1111/j.1525-1497.2005.40135.x.

11. Burke RE, Jones CD, Hosokawa P, Glorioso TJ, Coleman EA, Ginde AA. Influence of nonindex hospital readmission on length of stay and mortality. Med Care. 2018;56(1):85-90. https://doi.org/10.1097/MLR. 0000000000000829.

12. McAlister FA, Youngson E, Kaul P. Patients with heart ailure readmitted to the original hospital have better outcomes than those readmitted elsewhere. J Am Heart Assoc. 2017;6(5):e004892-e004897. https://doi. org/10.1161/JAHA. 116.004892.

13. Flaks-Manov N, Shadmi E, Bitterman H, Balicer R. Readmission to a different hospital-risk factors and impact on length of stay. Value Health. 2014;17(3):A148. https://doi.org/10.1016/j.jval.2014.03.860.
14. Galanter WL, Applebaum A, Boddipalli V, et al. Migration of patients between five urban teaching hospitals in Chicago. J Med Syst. 2013;37(2):1989-8. https://doi.org/10.1007/s10916-0139930-y.

15. Graboyes EM, Kallogjeri D, Saeed MJ, Olsen MA, Nussenbaum B. Postoperative care fragmentation and thirty-day unplanned readmissions after head and neck cancer surgery. Laryngoscope. 2016;127(4):868-874. https://doi.org/10.1002/lary.26301.

16. Chappidi MR, Kates M, Stimson CJ, Bivalacqua TJ, Pierorazio PM. Quantifying nonindex hospital readmissions and care fragmentation after major urological oncology surgeries in a nationally representative sample. J Urol. 2017;197(1):235-240. https://doi.org/10.1016/j.juro.2016.07. 078.

17. Parreco J, Buicko J, Cortolillo N, Namias N, Rattan R. Risk factors and costs associated with nationwide nonelective readmission after trauma. J Trauma Acute Care Surg. 2017;83(1):126-134. https://doi.org/10.1097/ TA.0000000000001505.

18. Brooke BS, Goodney PP, Kraiss LW, Gottlieb DJ, Samore MH, Finlayson SRG. Readmission destination and risk of mortality after major surgery: an observational cohort study. Lancet. 2015;386(9996):884-895. https://doi.org/10.1016/S0140-6736(15) 60087-3.

19. Justiniano $\mathbf{C F}, \mathbf{X u} \mathbf{Z}$, Becerra $\mathbf{A Z}$, et al.Long-term deleterious impact of surgeon care fragmentation after colorectal surgery on survival. Dis Colon Rectum. 2017;60(11):1147-1154. https://doi.org/10.1097/DCR. 0000000000000919.

20. Kothari AN, Loy VM, Brownless SA, et al. Adverse effect of postdischarge care fragmentation on outcomes after readmissions after liver transplantation. J Am Coll Surg. 2017;225(1):62-67. https://doi.org/10. 1016/j.jamcollsurg.2017.03.017.

21. Stang A. Critical evaluation of the Newcastle-Ottawa scale for the assessment of the quality of nonrandomized studies in meta-analyses. Eur J Epidemiol. 2010;25(9):603-605. https://doi.org/10.1007/s10654010-9491-z.

22. Wolinsky FD, Miller TR, An H, Brezinski PR, Vaughn TE, Rosenthal GE. Dual use of Medicare and the Veterans Health Administration: are there adverse health outcomes? BMC Health Serv Res. 2006;6(1):651-11. https://doi.org/10.1186/1472-6963-6-131.

23. Jia H, Zheng Y, Reker DM, et al. Multiple system utilization and mortality for veterans with stroke. Stroke. 2007;38(2):355-360. https:// doi.org/10.1161/01.STR.0000254457.38901.fb.

24. Hua M, Ng Gong M, Miltiades A, Wunsch H. Outcomes after rehospitalization at the same hospital or a different hospital following critical illness. AJRCCM. 2017;195(11):1486-1493.

25. Zheng C, Habermann EB, Shara NM, et al. Fragmentation of care after surgical discharge: non-index readmission after major cancer surgery. J Am Coll Surgeons. 2016;222(5):780-789.e782. https://doi.org/10.1016/ j.jamcollsurg.2016.01.052.

26. Saunders RS, Fernandes-Taylor S, Kind AJH, et al. Rehospitalization to primary versus different facilities following abdominal aortic aneurysm repair. J Vasc Surg. 2014;59(6): 1502-1510.e1502. https://doi.org/10. 1016/j.jvs.2013.12.015.

27. Glebova NO, Hicks CW, Taylor R, et al. Readmissions after complex aneurysm repair are frequent, costly, and primarily at nonindex hospitals. J Vasc Surg. 2014;60(6): 1429-1437. https://doi.org/10.1016/j.jvs. 2014.08.092.

28. Staples JA, Thiruchelvam D, Redelmeier DA. Site of hospital readmission and mortality: a population-based retrospective cohort study. CMAJ Open. 2014;2(2):E77-E85. https://doi.org/10.9778/cmajo.20130053.

29. Tsai TC, Orav EJ, Jha AK. Care Fragmentation in the postdischarge period. JAMA Surg. 2015;150(1):59-6. https://doi.org/10.1001/ jamasurg.2014.2071.

30. Pak JS, Lascano D, Kabat DH, et al. Patterns of care for readmission after radical cystectomy in New York State and the effect of care fragmentation. Urol Oncol. 2015;33(10):426.e13-426.e19. https://doi. org/10.1016/j.urolonc.2015.06.001.

31. Stitzenberg KB, Chang Y, Smith A, Meyers MO, Nielsen ME. Impact of location of readmission on outcomes after major cancer surgery. Ann Surg Oncol. 2017;24(2):319-329. https://doi.org/10.1245/s10434-0165528-1.

32. Luu N-P, Hussain T, Chang H-Y, Pfoh E, Pollack CE. Readmissions after colon cancer surgery: does it matter where patients are readmitted? JOP. 2016; 12(5):e502-e512. https://doi.org/10.1200/JOP.2015.007757.

33. Flaks-Manov N, Shadmi E, Hoshen M, Balicer R. Health information exchange systems and length of stay in readmissions to a different hospital. J Hosp Med. 2016;11(6):401-406. 
34. Axon RN, Gebregziabher M, Everett CJ, Heidenreich P, Hunt K. Dua health care system use is associated with higher rates of hospitalization and hospital readmission among veterans with heart failure. Am Heart J. 2016;174(C):157-163. https://doi.org/10.1016/j.ahj.2015.09.023.
35. Haggerty JL, Reid RJ, Freeman GK, Starfield BH, Adair CE, McKendry R. Continuity of care: a multidisciplinary review. BMJ. 2003;1219-1221.

Publisher's Note Springer Nature remains neutral with regard to jurisdictional claims in published maps and institutional affiliations. 